\title{
On Scalability and Mobility Management of Hierarchical Large-Scale Ad Hoc Networks
}

\author{
Ming-Hui Tsai ${ }^{2}$, Tzu-Chiang Chiang ${ }^{1,3}$, and Yueh-Min Huang ${ }^{1}$ \\ ${ }^{1}$ Department of Engineering Science, \\ National Cheng-Kung University, Taiwan, R.O.C \\ huang@mail.ncku.edu.tw \\ ${ }^{2}$ National Tainan First Senior High School, Taiwan, R.O.C. \\ coluxatnfsh.tn.edu.tw \\ ${ }^{3}$ Department of Information Management, \\ Hisng-Kuo University of Management, Taiwan, R.O.C. \\ n9892105@mail.ncku.edu. tw
}

\begin{abstract}
With the increased interest in the mobile wireless communication, the development of large-scale ad hoc networks has drawn a lot of attention and the scalability of ad hoc networks has been the subject of extensive research. The ad hoc network topology changes frequently and unpredictably, and mobility become extremely challenging in the circumstance. So, the broadcast storm becomes a very serious problem to migrate into such networks for the applications of group communications. The main concept of virtual subnet technology is the capability to group users into broadcast domains, which divides a virtual subnet into logic, instead of physical, segments and reduces the traffic overhead. With this characteristic, we propose an interoperability network model integrating self-organizing ad hoc networks and the Internet/a conventional network with the partition of physical/virtual subnets. Moreover, we describe a protocol to establish the virtual broadcast domains by using the IPv6 addressing concept in ad hoc networks and perform IP-based network communications in a multi-switch backbone. The hierarchical networks, physical/virtual subnets, addressing method and mobility management are described, and some performance issues are evaluated.
\end{abstract}

\section{Introduction}

Mobile ad hoc networks need the flexibility to collect more than two devices equipped with wireless communication and networking capability. Many handhelds, PDAs, laptops, notebooks and even mobile phones now also include wireless connectivity as a standard feature, and can access the Internet by a central system administration (like base stations in a cellular system or access-points in a wireless LAN) anytime, anywhere $[4,5,11,12]$. However in some circumstances a group of people makes up a party, a conference, or participates a camping site, and there has been a growing interest in rapidly deployable and dynamically reconfigurable wireless network supporting multimedia communication (data, voice and video) not just voice as mobile phone does. Each node acts as a router and is responsible for 
dynamically discovering other nodes which it can directly communicate with $[6,7,8$, 9]. The network topology of an ad hoc network changes frequently and unpredictably, so scalability and mobility become extremely challenging in the circumstance we mentioned previously. For many multimedia applications, however, it is desired that an inter-working functionality between the protocols in the ad hoc network and the IP-based Internet. Ad hoc networks should be adapted to deploy rapidly and can freely communicate with the traditional mobile wireless networking systems without the support of the pre-existing network infrastructures $[1,2,3,10]$.

However, the broadcast storm becomes a very serious problem for ad hoc networks to migrate into such networks for the application of group communications. The main concept of virtual subnet technology is the capability to group users into broadcast domains, which divides a network into logic, instead of physical, segments and reduces the traffic overhead. The network-layer address is used to inform the router physical segment where must send data to. While clients and servers may be distributed anywhere on a network, they are grouped together by virtual subnet technology, and broadcasts are sent within the same virtual subnet $[16,17,18]$.

Previous work on mobile radio networks with heterogeneous communication and dynamically changing topology concentrated primarily on mobility and/or broadcast storm problems in arbitrary geographical topologies. To solve mobility problem and improve network performance and signaling/control overhead in such a heterogeneous scenario were proposed. More recently, the issues of cluster backbone and multi-cluster architecture for mobile ad hoc networks were also proposed [13, 14, $15,19]$.

This paper addresses the interworking between ad hoc networks and Internet Protocol (IP-based) networks, where we focus on network partitioning can improve or solve critical functions such as mobility management, routing and broadcast problems. It can be observed in this type of architecture using network partitioning concept may result in larger scalability. To achieve this network interconnection using partitioning network, this heterogeneous communication establishes the installation of gateways with the help of specific access routers, which understand the both protocols of the ad hoc network and the IP suite. We also discuss an architecture based on a specific logical topology by partitioning a mobile ad hoc network into logically independent subnets.

\subsection{Research Contributions}

We consider a core network of 802.1Q-liked switches interconnected by trunk lines in ad hoc networks, and this network can span one or more small towns in sparsely populated areas, interconnecting communities as well as company LANs. Our intent is to provide both telephony and data services over the same technology (Ethernet), stack (IP), with seamless integration with the Internet. Our work chooses to distribute the bandwidth according to a hierarchical link sharing model.

The rest of this paper is organized as follows. We describe the problem for this network model in Section 2. Section 3 addresses in detail for the infrastructure of the scalable environment with ad hoc networks, Section 4 performance simulations with NS2 module are described. Finally, section 5 provides our concluding remarks and future works. 


\section{Problem Description}

With the fast development of wireless technology, the ad hoc network is walking out from research papers and becoming closer to the common consumers. A large-scale network issue will bring ad hoc networks a new future for distributed communication and computing. The motivation behind our approach is that network partitioning can improve some critical functions as broadcast storm problems defined such channel access, routing, mobility management and scalability, while reducing signaling/ control overhead within a hierarchical network.

Hence broadcasting in a shared wireless medium may result in multiple nodes receiving a transmission destined for a single node, and ultimately, in multiple transmission mutually interfering at a single node. Nodes can reduce the chances of interference by separating transmissions in time, space, frequency, or spreading code. By coordinating this separation instead of acting independently, nodes can further reduce the chances of interference and hence increase network throughput. The partition-based control structure provides a natural organization of network nodes that simplifies coordination of transmission among neighboring nodes by geographic area. It can be observed in this type of network that portioning may result also in lower congestion and high throughput compared to one large network.

This paper also discusses a network hierarchical architecture based on a specific logical topology superimposed over a physical topology (determined by transmission coverage of network nodes in the same geographic area); the architecture selects links to be affiliated (logical links) with physical links. Our main concern is finding an efficient logical topology and a suitable routing procedure which result in high performance and node mobility. In this architecture, network nodes of a cluster ad hoc network are grouped into two types of subnets: physical and virtual, and may dynamically change their affiliation with these subnets due to their mobility. Each node is allocated an address based on its current subnet affiliation and associated by IPv6 addressing. We consider networks that have several tens to several hundreds mobile nodes within this hierarchical network. We assume that there exists a channel access protocol which resolves contentions and/or interference in the network.

\subsection{Network Partitioning}

In the flat ad hoc network, all nodes participating in are assumed to be equal and share the same radio capacity. It has been proven that the flat network scheme works well in small size networks (e.g., fewer than 100 nodes) [20, 21]. However, when wireless network size increase (beyond certain thresholds), it becomes infeasible because of link and processing overhead. Building a physical hierarchical structure ad hoc network is a very promise way to solve the scalability problem. The most popular way of building hierarchy is to partition the networks into some clusters or group nodes geographically close to each other into clusters.

There are several approaches to construct the cluster; these include (1) Lowest-ID Cluster Algorithm (LID) [22], (2) Highest-Connectivity Cluster Algorithm (HCC) [23], (3) Least Clusterhead Change Algorithm (LCC) [24], (4) Distributed Clustering Algorithm (DCA) [25], and (5) Weighted Clustering Algorithm (WCA), [26]. The existing clustering algorithms differ on the criterion for the selection of their 
clusterheads. Once a node becomes the clusterhead, neither that node nor its members can participate in the cluster election algorithm. The election algorithm will terminate once all the nodes either become a clusterhead or a member of a clusterhead.

\section{Architecture of a Hierarcical Large-Scale Network}

As shown in Figure 1, four groups of mobile nodes form an internetworking wireless ad hoc network with IP-based Internet. The traditional ad hoc networks do not access the Internet or wired networks. However, due to the rapidly grow of the $3 \mathrm{G}$ communication and mobile devices, the hierarchical mobile communication architectture will be a new trend in the future. The communication between each node in this network infrastructure is established by using agent multi-route equipments and wireless multi-hop paths. By using this architecture, some mobile nodes (MNs) in this ad hoc network can access the Internet or communicate with ad hoc nodes of different wireless groups. Some critical issues for these MNs are their mobility management, the overhead and the load analysis for the agents to adapt well to dynamic topology changes by partitioning a mobile network into logically independent virtual subnets.

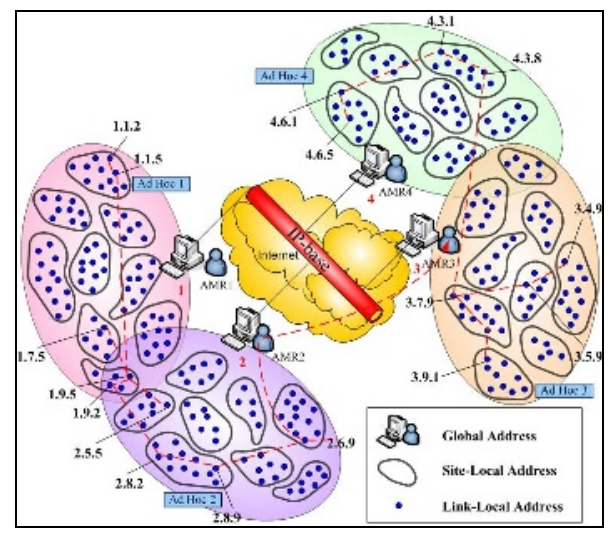

Fig. 1. An internetworking wireless ad hoc network with IP-based Internet (including Routing Path)

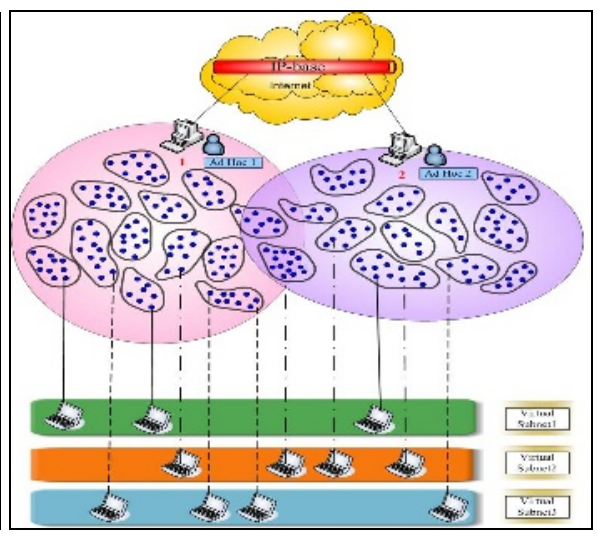

Fig. 2. An IP-based Internet with two ad hoc network groups and three virtual subnets

Hence mobile nodes may change their affiliation of physical and virtual subnets due to their mobility. First, in order to access the IP-based Internet and form the physical/virtual subnet, a set of Agent Multi-Route Routers (AMRs) is established in advance. The AMRs are connected to the Internet part as a gateway and communicate with the mobile nodes in wireless ad hoc networks via wireless transceivers. Each AMR consists of three components:

1.) IP-based component with traditional IP-based protocol suite installed is designed to connect with IP-based Internet (either wired or wireless); 
2.) Ad hoc component with ad hoc related protocols installed is connected with ad hoc networks through a wireless interface;

3.) Virtual subnet core component provide the intelligence to make filtering and forwarding decisions with configuration tables. Virtual subnet technology allows the nodes of different ad hoc network groups connected to form a virtual subnet, as Figure 2 depicted.

\subsection{Addressing Autoconfiguration with Physcal/Virtual Subnet Concepts}

We specify a mechanism by which mobile nodes in an ad hoc network may autoconfigure IPv6 addresses depending on their current physical and logical connectivity and address availability which make the IP address unique throughout the connected portion of the hierarchical network. Since Ipv6 defines both a stateful and stateless address autoconfiguration to enable plug-and-play networking of devices and reduce administration overhead. In general, each mobile node may autoconfigure a link-local address first and verifying its uniqueness on a link by using the duplicate address detection, and determine what information should be autoconfigured (addresses, other information, or both). However, in order to speed the autoconfiguretion process a mobile node may generate its link-local address in parallel with waiting for a Router Advertisement to generate a site-local and/or global address.

In order to improve the scalability of our proposed network, this hierarchical network is segmented into two parts, $x$ groups of ad hoc networks and an IP-based Internet. Assume that each group of ad hoc networks is segmented into y physical subnets each containing up to $\mathrm{z}$ mobile nodes. We describe the address space over an alphabet of size $\mathrm{m}=\max (\mathrm{x}, \mathrm{y}, \mathrm{z})$ containing the numbers $0,1,2, \ldots \mathrm{m}-1$. Each mobile node in the cluster ad hoc networks is given a triple number, where the global significant digit (GSD) is a digit in IP-based, the site significant digit (SSD) is a digit in site-local-based and the link significant digit (LSD) is a digit in link-local-based. Therefore, the total number of mobile nodes possible is $\mathrm{N}=$ xyz. The partitions of these groups are the basic building blocks and the Ipv6 address autoconfiguration process of the network. The address of a mobile node is separate to two: network prefix, which identifies ad hoc group networks and physical/virtual subnet ID, which identifies the affiliations of mobile nodes. Physical/virtual subnet ID is configured by the node on its current subnet affiliation as a result of its logical-topology or logicallink position relative to other mobile nodes, and prefix is determined by the AMR.

\subsection{Node Affiliations}

Because ad hoc networks do not allow the aggregation techniques especially about scalability issue that are available to traditional IP-based routing protocols. In general, loss of aggregation results in bigger route table for proactive protocol or longer delay for reactive protocol. Our node affiliations maintain aggregation for ad hoc networks and involve in a physical subnet which nodes are close in a local geographical area and a virtual subnet which nodes form a logical network. Figure 2 also shows a hierarchical network with the aggregation concept of physical subnets and virtual subnets. We use the GSD to present a cluster ad hoc network group that all nodes in this group partition into physical subnets with the same SSD and virtual subnets with the same LSD. Therefore in this hierarchical network architecture, network nodes are grouped into 
three types of clusters/ or subnets which mobile nodes may dynamically change their affiliation with these subnets according to their mobility. Members of different physical subnets are clustered together to form virtual subnets, each of which ideally spans all physical subnets and is used to provide communication paths among distant nodes.

\subsection{Routing}

Using hierarchical routing, the groups of ad hoc networks are physically and logically separated into different subnets. First, we describe a shortest path routing procedure. When a mobile node receives a packet, it checks the network prefix and the physical/virtual subnet ID. If it is the destination, it stops the relay process and operates the packet. If the mobile node is not the destination, the communication in this heterogeneous environment can be categorized into two scenarios:

Communication among ad hoc mobile nodes across the Internet. If a mobile node wants to communicate with the mobile nodes across the Internet, it knows from the network prefix (GSD) of the destination address that this destination belongs to different AMR. In this procedure routes traverse one digit at a time in the same physical subnet. For example, when the address of the source is 2.8 .2 and the address of destination is 3.4.9, this procedure would use the routing path $2.8 .2 \rightarrow 2.8 .9 \rightarrow$ 2.6.9 $\rightarrow \operatorname{AMR}(2) \rightarrow \operatorname{AMR}(3) \rightarrow 3.7 .9 \rightarrow 3.5 .9 \rightarrow 3.4 .9$. Once the AMR receives the forwarding packets, it sends them to the AMR of the destination across the Internet.

Communication among ad hoc mobile nodes in overlay ad hoc groups. This routing is performed in two phases. In the first phase routing is performed as the previous scenarios, Communication among ad hoc mobile nodes across the Internet. In the second phase packets are routed between the two connected ad hoc groups, where packets are routed inefficiently in strict hierarchical routing by using AMR gateways. Figure 1 gives an example for path selection in this network scenario. Two mobile nodes, 2.5.5 and 1.7.5, belong to two different ad hoc clusters located in a connected ad hoc network. Nodes 1.7.5 and 2.5.5 select AMR1 and AMR2 as a default gateway and use their network prefix respectively. If we use the hierarchical path which forwards the packets from AMRs instead of using the direct routing from these two different ad hoc clusters, a path selection function will be involved to choose a better routing between the hierarchical and flat routing.

\subsection{Mobility Management}

Each node in the cluster ad hoc network is affiliated with a physical subnet (SSD group) and use LSD group to form a virtual subnet. In order to connect the IP-based Internet communication, the mobile nodes need to discover the existence of the AMRs, which it belongs to, and join one of the AMRs first. The mobile nodes also must configure an IP address with the prefix of a reachable AMR (GSD group). With this partition-dependent address each mobile node can communicate with either the nodes in the same cluster network or the nodes through the Internet described as the above. The AMRs discovery could be done either by listening to an AMR Advertisement sent by AMRs (passive method) or sending an AMR REQUEST message sent by a mobile node (active method) at the initial state. In practice, both discovery methods can be combined and run in parallel. The AMR periodically sends 
out advertisement, and all nodes in its radio range store this information. An AMR REQUEST, which was broadcasted by a mobile node that is not in the radio range of an AMR, can now be answered by any intermediate node with stored AMR information, thus reducing the signalling traffic. After successful AMR discovery, the mobile node registers with one of the discovered AMRs.

A mobile node which changed its subnet affiliation will notify all the nodes in its new physical and virtual subnets of its newly acquired address. We can classify two types of subnet affiliations of mobility management.

(1) Mobility within the same cluster ad hoc networks:

After moving to a new location within the same cluster a mobile node ( $\mathrm{MNa}$ ) notifies its logical neighbors and the originated AMR by sending a location update (loc_update). Another mobile node $(\mathrm{MNb})$ which desires to communicate with the $\mathrm{MNa}$ will inquire at its logical neighbors about $\mathrm{MNa}$ by sending a location inquiry (loc_inquire). At least one of MNb's logical neighbors is also MNa's logical neighbor, thus, one of their mutual logical neighbors will provide $\mathrm{MNb}$ with $\mathrm{MNa}$ 's address by sending loc_track. After tracking down MNa's address, $\mathrm{MNb}$ sends its data to $\mathrm{MNa}$ via one of their mutual logical neighbors. If $\mathrm{MNb}$ is out of the cluster, the routing path will be updated while the MNa's loc_update received by MNa's originated AMR.

(2) Mobility within the different cluster ad hoc networks:

Each MN has a permanent IPv6 home address, which has the prefix of its home network and serves as a consistent and unique identifier for the MN. While this MN moves far away from the originated register AMR and loses the connection with the members of its subnet affiliation, it may participate in the new cluster and configure a new CoA. Note that it could not only connect with its new physical and virtual subnet members but also aware of the location of this new AMR in this new cluster. Finally it sends out a BINDING UPDATE message across Internet through the new AMR to its originated register AMR (serving as Home Agent). The home agent stores the mapping between an MN's home address and CoA in a so-called binding cache and acts as a proxy for the MN. Packets addressed to a node's home address are received by the home agent and forwarded (tunneled) to the MN's CoA according to the mapping information.

Whenever a mobile node moves to another cluster and has to select a different AMR, it should become a new number of physical and virtual subnets of selected AMR, and configure a new IP address with the new prefix. Thus, when registers with a certain AMR, mobile node generates an IP care-of-address (CoA) with the IP prefix of the selected AMR. After the switch to the new AMR is completed, the mobile node generates a new IPv6 CoA with the new network prefix. The AMR can be regarded as the default gateway for virtual subnet. In other words, the entire ad hoc network is logically separated into several virtual subnets.

Unicast routing in the ad hoc side is operated in a hierarchical way based on the subnet partitioning, i.e. packets addressed to a destination in the same subnet can be directly forwarded, while packets address to a destination in a different subnet must be routed through the AMRs (even if there is a direct wireless multi-hop link between the correspondent nodes). 


\section{Simulation Analysis}

To demonstrate the benefits of our adapted techniques, numerous simulations of our proposed protocol have been performed using the NS2 simulation which we enhanced the ability for mobile ad hoc nodes having communication with Internet. We also added $\mathrm{C}++$ Partition Toolkit (CCPT) to determine the physical and virtual subnets to partition the nodes in a cluster ad hoc network of our proposed hierarchical network. The mobility model used in each of the simulations is in a random direction. In each ad hoc network group, nodes are initially placed randomly within a predefined $500 \mathrm{~m}$ x $500 \mathrm{~m}$ grid area. Each node then chooses a random direction between 0 and 360 degrees and a speed from 0 to 20 meter/second.

First, we compare the efficiency of the proposed method to Flooding, AODV, and DCA with our $\mathrm{C}++$ Partition Toolkit. There are $\mathrm{n}(\mathrm{n}=\mathrm{x} * \mathrm{y} * \mathrm{z})$ nodes in our simulation grid area with $\mathrm{x}$ ad hoc network groups, $\mathrm{y}$ physical subnets and $\mathrm{z}$ virtual subnets and nodes can communicate each other within transmission range. Each physical subnet and virtual subnet is formed with randomly members. In the simulation study, we vary $\mathrm{x}, \mathrm{y}$ and $\mathrm{z}$ to compare how the network density and physical/virtual subnet sizes affect the efficiency.
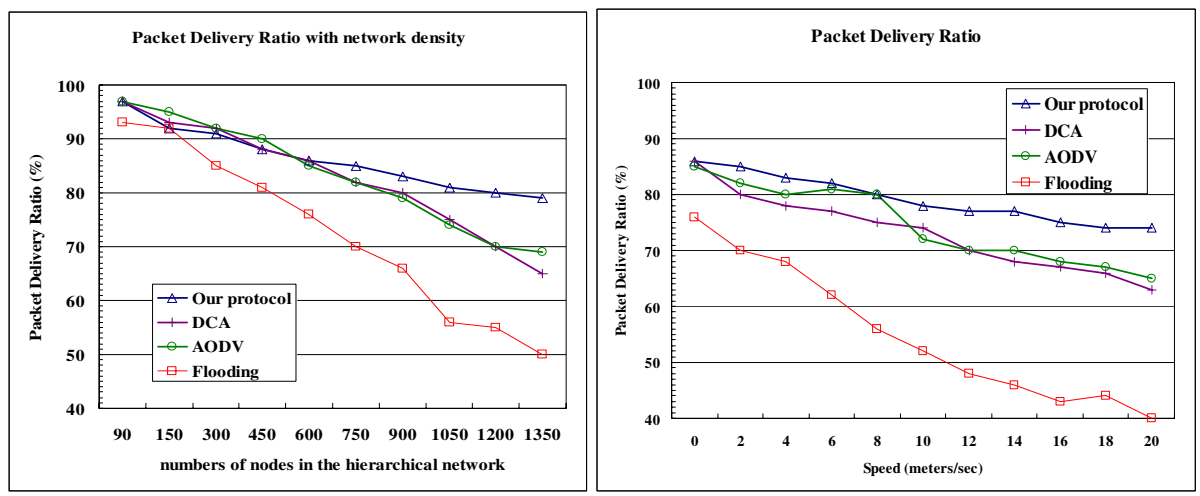

Fig. 3. The percentage of successful deliver Fig. 4. Packet delivery ratio with different speed packets

Figure 3 shows the result for network sizes from 90 nodes to 1350 nodes with 3 to 45 virtual subnets in a fixed 3 ad hoc network groups and 10 physical subnets. As a consequence of the greater node density, our proposed method is more efficient to deliver packets to all nodes in this hierarchical network. There are some nodes moving out of transmission range, which cannot re-route successfully. It shows that the packet delivery percentage up to $78 \%$ in a dense network with 45 subnets by our proposed method. In the same condition, AODV and original flooding achieves only $69 \%$ and $50 \%$. The main impact of the improvement is that our proposed method reroutes successfully by virtual subnets before nodes moving out of transmission range, but original flooding has too many redundant transmissions to reach destination just in time. 
Figure 4 refers to a 600-node ad hoc network with 3 groups, 10 physical subnets and 20 virtual subnets. It shows the percentage of successful multicast deliver ratio for mobile nodes whose velocity varies from $0 \mathrm{~m} / \mathrm{s}$ to $20 \mathrm{~m} / \mathrm{s}$ (around $70 \mathrm{~km} / \mathrm{h}$ ) using four different routing protocols, and all the nodes of the addressed multicast group received the packet more than $85 \%$ when the velocity is less than $8 \mathrm{~m} / \mathrm{s}$ for our proposed method. However, when the velocity of nodes is more than $2 \mathrm{~m} / \mathrm{s}$, the packet delivery ratio of flooding will drop immensely as the velocity of nodes speed up.

\section{Conclusions and Future Research}

In this paper, we have described the adapted partition network model for large-scale ad hoc networks. The main objective of our protocol is to perform efficiently the behaviours of virtual subnets in ad hoc networks. We propose an interoperability network model integrating a self-organizing ad hoc network and the Internet/a conventional network with the same virtual subnet. Moreover, we describe a protocol to establish the virtual broadcast domains by using the IPv6 multicast-membership in ad hoc networks and perform IP-based network communications in a multi-switch backbone. Since the partition technology functions by logically segmenting the network into different broadcast domains, packets can only be delivered between fixed/mobile nodes with the same physical/virtual identity (group member). Therefore we can prevent the broadcast storm problem in MANET.

We plan to identify the suitable cache table refreshing mechanism on the proposed method in the future works. We will also generalize the clustering method to progress the behaviours of virtual subnets so that they can be applied in mobile ad hoc networks.

\section{References}

1. Gupta and D. Ferrari, (1995), Resource Partitioning for Real-time Communication, IEEE/ACM Trans. on Networking, 3.5, 501-518.

2. N. Kavak, (1995), Data Communications in ATM Networks, IEEE Network, vol.9, no.3,May/June 1995.

3. Rajaravivarma, V.; (1997), Virtual local area network technology and applications, Proceeding of the Twenty-Ninth Southeastern Symposium on ,9-11 March 1997 Pages: 49 - 52 .

4. Internet Engineering Task Force (IETF) Mobile Ad Hoc Networks (MANET) Working Group Charter. http://www.ietf.org/html.charters/manet-charter.html.

5. J. Jubin and J.D. Tornow, "The DARPA Packet Radio Network Protocols," Proceeding of the IEEE, Vol.75, no. 1, Jan. a987, pp. 21-32.

6. E. Bommaiah, M. Liu, A. McAuley, and R. Talpade, “AMRoute: Adhoc Multicast Routing Protocol," Internet-Draft, draft-talpade-manet-amroute-00.txt, Aug. 1998, Work in progress.

7. C. W. Wu, Y.C. Tay, and C.-K. Toh, "Ad hoc Multicast Routing protocol utilizing Increasing id-numberS (AMRIS) Functional Specification," Internet-Draft, draft-ieftmanet-amris-spec-00.txt, Nov. 1998, Work in progress. 
8. J.J. Garcia-Luna-Aceves and E.L. Madruga, “Core-Assisted Mesh Protocol,” IEEE Journal On Selected Areas in Communications, Vol. 17, no. 8, Aug. 1999, pp. 784-792.

9. D. J. Baker and A. Ephremides, (1981), The architectural organization of a mobile radio network via a distributed algorithm, IEEE Trans.Commun., pp. 1694-1701, Nov. 1981

10. D. J. Baker, J. Wieselthier, and A. Ephremides, (1982), A distributed algorithm for scheduling the activation of links in a self-organizing, mobile, radio network, in Proc. IEEE ICC'82, pp. 2F.6.1-2F.6.5.

11. I. Chlamtac and S. S. Pinter, (1987), Distributed nodes organization algorithm for channel access in a multihop dynamic radio network, IEEE Trans.Comput., pp. 728-737, June 1987.

12. IEEE Std 802.1Q, 2003 Edition. (2003), IEEE standards for local and metropolitan area networks, Virtual bridged local area networks.

13. Tzu-Chiang Chiang, Ching-Hung Yeh and Yueh-Min Huang,(2004) A Forwarding Cache VLAN Protocol (FCVP) in wireless Networks, 4th IASTED International MultiConference on Wireless and Optical Communications - WOC 2004, July 8-10, 2004,Banff,Canada

14. Wu, C., Tay, Y., and Toh, C.,(1998), Ad Hoc Multicast Routing Protocol Utilizing Increasing id-numbers (AMRIS) Functional Specification, Internet-Draft, Nov. 1998.

15. D.B. Johnson, C. E. Perkins, and J. Arkko, "Mobility support in IPv6." IETF internet draft, June 2002.

16. Jacob Sharony, "An architecture for mobile radio network with dynamically changing topology using virtual subnets", Mobile networks and applications, pp. 75- 86, 1996.

17. J. Xi and C. Bettstetter, "Wireless Multihop Internet Access: Gateway, Discovery, Routing and Addressing," in Proc. International Conference on $3^{\text {rd }}$ generation Wireless and Beyond, May 2002.

18. Jeffrey S. Chase, David E. Irwin, Laura E. Grit, Justin D. Moore, and Sara E. Sprenkle, "Dynamic Virtual Clusters in a Grid Site Manager", the 12th IEEE International Symposium on High Performance Distributed Computing, 2003.

19. Zhong Fana,, Siva Subramani, "An address autoconfiguration protocol for IPv6 hosts in a mobile Ad Hoc network", Computer Communications, Sep. 2004.

20. C. E. Perkins et al. "Ad hoc On-Demand Distance Vector (AODV) Routing," RFC 3561, July 2003, http://www.ietf.org/rfc/rfc3561.txt

21. D. B. Johnson and D. A. Maltz, "The Dynamic Source Routing Protocol for Mobile Ad Hoc Networks (DSR)," IETF Internet draft, 19 July 2004, http://www.ietf.org/internetdrafts/draft-ietf-manet-dsr-10

22. C.R. Lin, and M. Gerla, "Adaptive Clustering for Mobile Wireless Networks," IEEE Journal on Selected Areas in Communications, Vol. 15, No. 7, Sep. 1997, pp.1265-1275.

23. M. Gerla and J. T.C. Tsai, "Multicluster, Mobile, Multimedia Radio Networks," Wireless Networks vol.I , 1995, pp. 255-265.

24. C. C. Chiang, H. K. Wu, W. Liu and M. Gerla, "Routing in Clustered Multihop, Mobile Wireless Networks with Fading Channel," Proc. IEEE Singapore international Conference on Networks (SICON97), 1997, pp. 197-211.

25. S. Basagni, "Distributed Clustering Algorithm for Ad Hoc Networks," Proc. Int'l Symp. Parallel Architectures, Algorithms, and Networks (I-SPAN), June 1999, pp. 310-315.

26. M. Chatterjee, S.K. Das and D. Turgut, "WCA: A Weighted Clustering Algorithm for Mobile Ad hoc Networks", Journal of Clustering Computing, (Special Issue on Mobile Ad hoc Networks), Vol. 5, No. 2, April 2002, pp. 193-204. 